\section{Número ideal de filhos como fator de risco para laqueadura tubária}

\author{
Ideal number of children as a risk \\ factor for tubal ligation
}

\author{
${ }^{1}$ Faculdade de Ciências \\ Médicas, Universidade \\ Estadual de Campinas, \\ Campinas, Brasil. \\ 2 Centro de Pesquisa \\ Materno-Infantil de \\ Campinas, Campinas, Brasil. \\ Correspondência \\ J. G. Cecatti \\ Departamento de \\ Tocoginecologia, \\ Faculdade de Ciências \\ Médicas, Universidade \\ Estadual de Campinas. \\ Rua Alexander Fleming 101, \\ Campinas, $S P$ \\ 13081-970, Brasil. \\ cecatti@unicamp.br
}

\begin{abstract}
The purpose of this paper was to evaluate the association between ideal number of children (INC) and female sterilization. A nested casecontrol study was performed through a secondary analysis of data from a cohort study on the reproductive health of women in Campinas, São Paulo, Brazil. A total of 3,878 women were included, 1,012 being sterilized (cases). The relationship between INC and number of live births (LB) was divided in two categories (INC> $L B$ and $I N C \leq L B$ ). The relative risks of performing tubal ligation were calculated (odds ratio) with their respective 95\% confidence intervals for the relation INC/LB and all control variables. All predictor variables were included in a logistic regression model in order to identify the factors independently associated with female sterilization. The results showed that the risks of tubal ligation were higher among women with $I N C \leq L B$, higher age, with partners, higher family income, more than two previous pregnancies, more deliveries, fewer abortions, and without paid work.
\end{abstract}

Contraception; Tubal Ligation; Family Planning; Birth Control
Luiz Eduardo Campos de Carvalho 1 José Guilherme Cecatti 1 Maria José Duarte Osis 2 Maria Helena de Sousa 2

Introdução

O surgimento dos diferentes métodos anticoncepcionais (MAC) de alta eficácia, principalmente após 1960, contribuiu de forma determinante para a queda da fecundidade em todo o mundo 1 . No Brasil, segundo Perpétuo \& Aguirre 2 , a difusão dos diversos MAC contribuiu sobremaneira para a queda da fecundidade, inicialmente nos grandes centros urbanos e posteriormente em outras regiões do país. O reflexo dessa situação pode ser observado no rápido declínio da taxa de fecundidade total (TFT), que era de aproximadamente 6,0 em 1960 e passou para menos de 2,5 na década de 19903 .

Em todo o mundo, os governos, de um modo geral, sempre se preocupam com o crescimento populacional e com as políticas de regulação da fecundidade ou controle da natalidade. Os programas de planejamento familiar têm exercido um papel fundamental na diminuição da fecundidade, em especial nos países em desenvolvimento ${ }^{4}$. No Brasil, porém, até os meados da década de 80 , não houve programas de planejamento familiar, e o setor privado, por meio de cooperação com agências internacionais interessadas no controle da natalidade, atuou de forma livre e sem controle 5 . Nesse contexto, houve várias distorções na oferta de MAC, sendo a realização indiscriminada de esterilização cirúrgica feminina um dos principais abusos, chegando a constituir uma verda- 
deira indústria - a chamada "indústria da esterilização" 3 .

A partir de 1983, com o surgimento do Programa de Assistência Integral à Saúde da Mulher (PAISM), um novo conceito na abordagem da saúde feminina começou a ser difundido. Preconizava-se então uma "assistência integral" onde as ações deveriam ser ampliadas, e foi neste contexto que pela primeira vez incluiu-se um programa de planejamento familiar 6,7. Apesar disso, dadas as condições globais da saúde no país, esse programa não foi facilmente implementado e, em muitas regiões, nunca se consolidou 6. Um dos resultados desta situação é que apenas em 1997 foi aprovada a legislação que regulamenta a realização da laqueadura e da vasectomia no Brasil (Portaria no 144. Diário Oficial da União 1997; 24 nov), determinando, entre outras coisas, que a esterilização só pode ser feita depois que a (o) candidata (o) passe por sessões de aconselhamento em que sejam apresentadas todas as alternativas anticoncepcionais.

Neste cenário, nos últimos vinte anos, a prevalência da esterilização cirúrgica feminina continuou a crescer, e atualmente é o MAC mais prevalente entre as mulheres em idade fértil, como mostrou a Pesquisa Nacional sobre Demografia e Saúde (PNDS) de 1996, 8 onde $40,1 \%$ das mulheres unidas entre 15 e 49 anos estavam laqueadas. No Estado de São Paulo, o índice de laqueadura em 1996 era de 36,0\% (www. seade.gov.br/spmulher/, acessado em 04/Ago/ 2003).

Os motivos das mulheres para optarem pela laqueadura, geralmente incluem o não querer ter mais filhos, não poder ter mais filhos, não ter condições de criar mais filhos ou já haver alcançado o "número ideal de filhos" (NIF) 9,10,11. Entretanto, chama a atenção que as mulheres laqueadas têm um maior número de filhos do que as usuárias de métodos reversíveis, o que faz pensar que, provavelmente, quando chegam a optar, ou conseguem ter acesso à laqueadura, já tiveram mais filhos do que realmente desejariam ter tido 12,13 .

Por outro lado, um dos problemas relevantes gerados a partir da alta prevalência de laqueadura e de suas distorções é o aparecimento de um grande contingente de mulheres arrependidas de ter realizado a esterilização cirúrgica, sendo que a porcentagem varia de $10,0 \%$ a 20,0\% 9,11,14. É possível que essas mulheres sejam aquelas que gostariam de ter mais filhos, mas que em um dado momento de suas vidas não podem tê-los e, por falta de acesso a MAC reversíveis, acabam se submetendo à laqueadura precocemente, tornando-se candidatas ao arrependimento 15 .
Não foram encontrados na literatura nacional estudos que relacionem o arrependimento àquela que tem sido uma das principais justificativas das mulheres para optarem pela laqueadura: ter alcançado o NIF. Talvez, uma das razões para isto seja a dificuldade em operacionalizar o conceito de NIF. O que significa um número ideal de filhos? Esse número varia temporalmente? Como obter uma medida exata? Do ponto de vista da demografia, obter essa informação é essencial para explicar a tendência recente da fecundidade e avaliar a magnitude da fecundidade indesejada. As pesquisas do tipo Demographic Health Survey (DHS), sistematicamente incluem uma pergunta sobre esse assunto em seu questionário. Na última PNDS realizada no Brasil, em 1996 8, perguntou-se às mulheres entrevistadas: "Se você pudesse voltar à época em que não tinha nenhum filho e pudesse escolher quantos filhos gostaria de ter, quantos filhos teria?" Percebe-se que a formulação da pergunta procura situar de maneira clara o tempo e as condições em relação às quais as mulheres devem expressar sua preferência. Essa abordagem traz consigo, de fato, a possibilidade de obter respostas adaptativas, à medida em que existe um intervalo de tempo entre o momento em que se faz a pergunta e o momento em que aconteceu o nascimento dos filhos, e as condições atuais de vida das mulheres podem ter mudado, para melhor ou pior.

Em outros países, alguns estudos que tratam sobre a questão do NIF observam que, assim como em relação à fecundidade, nas últimas décadas do século XX houve uma queda do tamanho ideal de família. Jejeebhoy 16, em Taiwan, aponta que o NIF era de 4,0 entre os anos de 1965-1970 e passou para 2,9 no ano de 1976. Herrera 17, de forma semelhante, descreve que também no México o NIF diminuiu de 4,5 para 3,3 entre 1976 e 1987. No Brasil, em 1986 verificou-se que o NIF médio era de 2,8 18, enquanto os resultados da PNDS de 19968 evidenciaram que o NIF médio das entrevistadas foi de 2,3.

Bongaarts 1 sugere uma explicação para esse fenômeno, afirmando que, com o advento do desenvolvimento econômico dos países, o custo para se manter uma criança superaria os benefícios de tê-las. Martine 3 e Perpétuo \& Aguirre 2, apesar de não tratarem especificamente da queda do NIF, mas sim da queda de fecundidade no Brasil, sugerem que os efeitos da pressão econômica fizeram com que as pessoas sentissem uma maior necessidade de controlar mais efetivamente a sua natalidade. Entretanto, no caso das mulheres que optam pela laqueadura no Brasil, existe uma interrogação 
quanto à legitimidade de se afirmar que essa opção seja decorrente delas terem atingido o NIF, uma vez que, em geral, a sua média de filhos vivos é maior do que a das usuárias de MAC reversíveis 13. Além disso, dados nacionais de 198618 e de 19968 também indicaram que, especialmente as mulheres com mais de três filhos - as potencialmente laqueadas, geralmente referiam um NIF menor do que o número de filhos observado. Nesse contexto, o objetivo deste artigo é correlacionar a prática da laqueadura com alguns fatores sócio-demográficos, dentre eles, especialmente o NIF.

\section{Sujeitos e métodos}

Foi elaborado um estudo de caso-controle aninhado a uma coorte, onde os dados utilizados foram coletados originalmente, em 1995, para a pesquisa $O$ impacto das altas taxas de cesariana sobre a fecundidade de uma população. Um estudo de coorte retrospectiva em Campinas, Brasil. Esse estudo originalmente incluiu as mulheres que tiveram seu primeiro filho em 1985, em Campinas, São Paulo, identificadas por intermédio das crianças freqüentando as escolas do município durante o ano de 1995, e então entrevistadas sobre aspectos sócio-demográficos, econômicos e de sua saúde reprodutiva. Das 4.252 mulheres inicialmente identificadas como elegíveis ao estudo, 3.878 (91,2\%) responderam o questionário, por meio de entrevistas realizadas num período de seis meses durante 1995, por uma equipe constituída de 24 pesquisadoras selecionadas e treinadas para esta finalidade, sob a supervisão de duas assistentes de pesquisa e uma coordenadora, responsáveis pela logística do trabalho de campo, verificação da consistência e confiabilidade das informações, através do controle de qualidade feito com a reaplicação de algumas entrevistas ao acaso 19. Do total de 3.878 mulheres entrevistadas, 1.012 estavam laqueadas e 2.866 não-laqueadas, sendo a prevalência acumulada de esterilização cirúrgica feminina nesta amostra de $26,1 \%$, e constituindo respectivamente os casos e controles para o desenho de estudo da presente análise.

Definiu-se como variável independente principal o NIF, pela comparação entre o número de filhos declarados pelas mulheres como ideal e o número de filhos nascidos vivos $(\mathrm{NV})$, resultando em duas categorias: NIF $>\mathrm{NV}$ e NIF $\leq$ NV, sendo a realização ou não de laqueadura a variável dependente. Para fins de análise estatística a primeira categoria foi tomada como referência. Todas as variáveis deste estu- do foram obtidas por meio das respostas ao questionário da pesquisa original. Avaliaramse, ainda, variáveis de controle sócio-demográfico: idade materna, escolaridade materna, estado marital, cor da pele (autodefinida pela entrevistada), trabalho remunerado (qualquer atividade remunerada fora ou no lar), renda familiar total (somatória das rendas de cada membro da família morando no mesmo domicílio, corte feito pela mediana) e as variáveis reprodutivas: número de gravidezes, número de partos, de abortos e de nascidos mortos.

Com base nos resultados da PNDS 1996, calculou-se o tamanho da amostra para estudo caso-controle 20 , utilizando a proporção de mulheres com NIF $\leq \mathrm{NV}$ igual a $45,0 \%$, como estimativa para a proporção de mulheres nãolaqueadas (controles) expostas ao fator de risco $(\mathrm{NIF} \leq \mathrm{NV})$. Para o odds ratio (OR) implicado de $1,3, \operatorname{com} \alpha$ igual a 0,05 e $\beta$ igual a 0,10 , o número de casos calculado foi de 917 mulheres e os controles 1.834, número inferior ao disponível no banco de dados.

Os riscos relativos estimados com intervalo de confiança (IC95\%) foram calculados usando inicialmente uma análise bivariada simples e, em seguida, estratificada (Mantel-Haenszel). Posteriormente, utilizou-se um modelo de regressão logística múltipla considerando a informação sobre laqueadura como variável dependente, e como variáveis preditoras, o NIF, idade da mulher, escolaridade, estado marital, cor da pele, trabalho remunerado, renda familiar, número de gestações, número de partos, de abortos e de nascidos mortos. O método de seleção utilizado para a análise da regressão foi o "forward", sendo que todas as variáveis foram consideradas no início, e aquelas que não constam no modelo final foram não-significativas.

Para a análise estatística foram utilizados dois pacotes estatísticos, SPSS e Epi Info. O projeto de pesquisa que sistematizou essa análise secundária de dados foi previamente aprovado pela Comissão de Pesquisa do Departamento de Tocoginecologia, Centro de Atenção Integral à Saúde da Mulher e pelo Comitê de Ética em Pesquisa da Faculdade de Ciências Médicas, Universidade Estadual de Campinas.

\section{Resultados}

Observa-se que somente não se verificou associação entre a opção pela laqueadura e a cor da pele, número de abortos e de nascidos mortos. A proporção de entrevistadas com menos de 35 anos foi menor entre as laqueadas $(57,2 \%) \mathrm{em}$ comparação com as demais $(72,2 \%)$. Em rela- 
ção à escolaridade, observou-se uma diferença de dez pontos porcentuais entre as laqueadas $\mathrm{e}$ as não-laqueadas que tinham até a 8 a série do ensino fundamental. Mais de 90,0\% das mulheres que haviam feito laqueadura tinham companheiro, comparado a $81,3 \%$ das não-laqueadas. A referência ao trabalho remunerado foi maior entre as entrevistadas não-laqueadas. A declaração de renda familiar superior a oitocentos Reais foi mais freqüente no grupo das laqueadas. A proporção de mulheres com mais de duas gravidezes e mais de dois partos foi significativamente maior entre as que haviam feito laqueadura (Tabela 1). O grupo das mulheres com NIF $\leq \mathrm{NV}$ apresentou um risco quase 2,5 vezes maior e significativo de estar laqueada do que as demais entrevistadas (OR bruto de 2,43 com IC95\% 1,98-2,98).

Controlando-se pelas variáveis sócio-demográficas estudadas, as mulheres com NIF $\leq$ NV mantiveram um maior risco de estarem laqueadas. Só não foi significativa a diferença no grupo de mulheres sem companheiro. Os maiores riscos, com OR acima de três, foram observados entre as mulheres com idade igual ou superior a 35 anos e as que referiram escolaridade acima da 8 a série do ensino fundamental. $\mathrm{O}$ risco de laqueadura esteve próximo de 2,5 entre as entrevistadas com companheiro, que se declararam brancas, que tinham trabalho remunerado e com renda acima de oitocentos Reais (Tabela 2).

Não se observou diferença estatística no risco de estar laqueada entre as mulheres com NIF $\leq \mathrm{NV}$ e que tinham mais de duas gravidezes, mais de dois partos e pelo menos um natimorto. Em geral o OR esteve entre 2,33, observado entre as mulheres com até dois partos, e 2,52, entre as que não declararam abortos (Tabela 3).

A Tabela 4 mostra os resultados do modelo de regressão logística múltipla, onde pode-se observar que as mulheres com maior idade, renda superior a oitocentos Reais, com companheiro, número de partos ou gestações superior a dois e com o NIF $\leq$ NV apresentaram um risco maior de estarem laqueadas no momento da entrevista. Por outro lado, o trabalho remunerado e a presença de um ou mais abortos apresentaram-se como fatores que diminuíram o risco de laqueadura.

\section{Discussão}

Os resultados apresentados sugerem que as mulheres com NIF $\leq \mathrm{NV}$, de uma maneira geral, apresentaram um maior risco de estarem laqueadas. Neste grupo de mulheres estariam aquelas com NIF < NV, que tiveram mais filhos do que desejariam, para as quais a laqueadura, provavelmente, representou a conseqüência direta de uma demanda insatisfeita de anticoncepção. Isso se deu em um contexto histórico e social em que, reconhecidamente, as mulheres no Brasil, de um modo geral, dispunham de poucas opções e enfrentavam dificuldades para ter acesso a métodos contraceptivos 3,5,6. Essa situação fez com que, a partir do final dos anos de 1970, a laqueadura se tornasse, freqüentemente, a única opção para mulheres que, em certa altura da vida reprodutiva, já teriam ultrapassado o número de filhos considerado ideal 13. Os dados da PNDS 1996 corroboram essa interpretação, pois apontam que, do total de nascidos vivos nos últimos cinco anos antes da pesquisa, mais de um quarto dos nascimentos não foram previstos e mais de $20,0 \%$ não foram desejados 8 .

Por outro lado, os resultados apresentados incluem como tendo maior risco de esterilizarem-se, também as mulheres com NIF $=\mathrm{NV}$, para as quais a laqueadura poderia significar uma opção legítima para regular a fecundidade, uma vez que o tamanho ideal de família teria sido alcançado. Entretanto, isto não pode ser aceito sem considerar pelo menos dois aspectos. Em primeiro lugar, a possível racionalização desenvolvida por muitas mulheres, que tendem a mencionar um NIF igual ao que tiveram, como uma forma adaptativa, ou seja, aumentam o NIF de acordo com o número de filhos tidos, pela dificuldade afetiva de admitir que não desejariam ter tido algum ou alguns deles 8,17,21. Em segundo lugar, não se pode afirmar que essas mulheres tenham optado pela laqueadura adequadamente, de maneira livre e informada, uma vez que o contexto em que se inseriam estava igualmente marcado pela falta de acesso e disponibilidade de opções contraceptivas, o que já foi mencionado acima. Outras pesquisas, com populações semelhantes às que fizeram parte deste estudo, indicaram que as mulheres que optavam pela esterilização cirúrgica, o faziam com base em informações de parentes e amigas, sem conhecer ou haver experimentado outros MAC 7,10,11,22,23.

Também é preciso considerar que o maior risco para a laqueadura dessas mulheres com $\mathrm{NIF} \leq \mathrm{NV}$, esteve associado a outras variáveis: a maior idade das mulheres entrevistadas, o fato de ter companheiro, a maior renda familiar e a presença de duas ou mais gestações. Essas associações também foram observadas por outros autores 11,12,24. Porém, ao contrário da maioria dos estudos, neste trabalho a escolaridade não se associou ao risco de laqueadura, o que 
Distribuição percentual das mulheres de acordo com características sócio-demográficas

e reprodutivas, segundo a realização ou não da laqueadura.

\begin{tabular}{|c|c|c|c|c|}
\hline Características & Laqueadas (\%) & Não-laqueadas (\%) & $\chi^{2}$ & $\mathrm{p}$ \\
\hline \multicolumn{5}{|l|}{ Idade (anos) } \\
\hline$<35$ & 57,2 & 72,1 & 76,4 & $<0,001$ \\
\hline$\geq 35$ & 42,8 & 27,9 & & \\
\hline \multicolumn{5}{|l|}{ Escolaridade } \\
\hline Até 8ạ série & 55,9 & 65,9 & 31,7 & $<0,001$ \\
\hline > 8ạ série & 44,1 & 34,1 & & \\
\hline \multicolumn{5}{|l|}{ Estado marital } \\
\hline Sem companheiro & 6,7 & 18,7 & 80,4 & $<0,001$ \\
\hline Com companheiro & 93,3 & 81,3 & & \\
\hline \multicolumn{5}{|l|}{ Cor/raça* } \\
\hline Cor/raça branca & 64,2 & 61,1 & 3,0 & $0,083(\mathrm{~ns})$ \\
\hline Outra cor/raça & 35,8 & 38,9 & & \\
\hline \multicolumn{5}{|l|}{ Trabalho } \\
\hline Não trabalha & 49,8 & 41,8 & 19,0 & $<0,001$ \\
\hline Trabalha & 50,2 & 58,2 & & \\
\hline \multicolumn{5}{|l|}{ Renda familiar ${ }^{\star \star}$} \\
\hline Até $\mathrm{R} \$ 800,00$ & 37,4 & 55,2 & 87,2 & $<0,001$ \\
\hline$>\mathrm{R} \$ 800,00$ & 62,6 & 44,8 & & \\
\hline \multicolumn{5}{|c|}{ Número de gravidezes } \\
\hline Até 2 & 46,8 & 65,9 & 113,9 & $<0,001$ \\
\hline$>2$ & 53,2 & 34,1 & & \\
\hline \multicolumn{5}{|l|}{ Número de partos } \\
\hline Até 2 & 62,5 & 79,5 & 114,9 & $<0,001$ \\
\hline$>2$ & 37,5 & 20,5 & & \\
\hline \multicolumn{5}{|l|}{ Número de abortos } \\
\hline Nenhum & 76,2 & 77,8 & 1,0 & 0,32 (ns) \\
\hline$\geq 1$ aborto & 23,8 & 22,2 & & \\
\hline \multicolumn{5}{|c|}{ Número de nascidos mortos } \\
\hline Nenhum & 98,8 & 99,0 & 0,04 & 0,849 (ns) \\
\hline$\geq 1$ aborto & 1,2 & 1,0 & & \\
\hline \multicolumn{5}{|l|}{ NIF versus NV } \\
\hline NIF > NV & 13,1 & 26,9 & 79,2 & $<0,001$ \\
\hline $\mathrm{NIF} \leq \mathrm{NV}$ & 86,9 & 73,1 & & \\
\hline Total de mulheres & 1.012 & 2.866 & & \\
\hline
\end{tabular}

* Falta informação de uma mulher; ** faltam informações de 278 mulheres; ns = não significativo. 
Distribuição percentual das mulheres segundo categorias de número ideal de filhos (NIF) versus número de nascidos vivos (NV), de acordo com características sócio-demográficas.

\begin{tabular}{|c|c|c|c|c|}
\hline NIF versus NV & Laqueadas (\%) & Não-laqueadas (\%) & $O R^{\star \star \star}$ & IC95\% por OR \\
\hline Idade (anos) & & & $2,62^{\star \star \star}$ & $2,16-3,27$ \\
\hline \multicolumn{5}{|l|}{$<35$} \\
\hline NIF $>$ NV & 12,3 & 23,5 & 1,00 & \\
\hline $\mathrm{NIF} \leq \mathrm{NV}$ & 87,7 & 76,5 & 2,19 & $1,66-2,89$ \\
\hline $\mathrm{n}$ & 579 & 2.068 & & \\
\hline \multicolumn{5}{|l|}{$\geq 35$} \\
\hline NIF > NV & 14,3 & 35,7 & 1,00 & $2,42-4,57$ \\
\hline $\mathrm{NIF} \leq \mathrm{NV}$ & 85,7 & 64,3 & 3,32 & \\
\hline $\mathrm{n}$ & 433 & 798 & & \\
\hline Escolaridade & & & $2,42^{\star \star \star}$ & $1,99-3,00$ \\
\hline \multicolumn{5}{|l|}{ Até 8ạ série } \\
\hline NIF > NV & 15,2 & 25,8 & 1,00 & $1,50-2,52$ \\
\hline $\mathrm{NIF} \leq \mathrm{NV}$ & 84,8 & 74,2 & 1,94 & \\
\hline $\mathrm{n}$ & 566 & 1.889 & & \\
\hline \multicolumn{5}{|l|}{ > 8a série } \\
\hline NIF > NV & 10,5 & 28,9 & 1,00 & $2,44-4,87$ \\
\hline $\mathrm{NIF} \leq \mathrm{NV}$ & 89,5 & 71,1 & 3,44 & \\
\hline $\mathrm{n}$ & 446 & 977 & & \\
\hline Estado marital & & & $2,36^{* \star *}$ & $1,92-2,91$ \\
\hline \multicolumn{5}{|l|}{ Sem companheiro } \\
\hline NIF > NV & 19,1 & 30,1 & 1,00 & $0,93-3,61$ \\
\hline$N I F \leq N V$ & 80,9 & 69,9 & 1,82 & \\
\hline $\mathrm{n}$ & 68 & 535 & & \\
\hline \multicolumn{5}{|l|}{ Com companheiro } \\
\hline NIF $>$ NV & 12,7 & 26,1 & 1,00 & $1,95-3,02$ \\
\hline $\mathrm{NIF} \leq \mathrm{NV}$ & 87,3 & 73,9 & 2,43 & \\
\hline $\mathrm{n}$ & 944 & 2.331 & & \\
\hline Cor da pele* & & & $2,43^{\star \star \star}$ & $1,98-2,98$ \\
\hline \multicolumn{5}{|l|}{ Branca } \\
\hline NIF > NV & 12,5 & 27,6 & 1,00 & $2,06-3,49$ \\
\hline $\mathrm{NIF} \leq \mathrm{NV}$ & 87,5 & 72,4 & 2,68 & \\
\hline $\mathrm{n}$ & 650 & 1.750 & & \\
\hline \multicolumn{5}{|l|}{ Outra cor } \\
\hline NIF $>$ NV & 14,4 & 25,7 & 1,00 & $1,47-2,88$ \\
\hline $\mathrm{NIF} \leq \mathrm{NV}$ & 85,6 & 74,3 & 2,06 & \\
\hline $\mathrm{n}$ & 362 & 1.115 & & \\
\hline Trabalho & & & $2,40^{\star \star \star}$ & $1,95-2,94$ \\
\hline \multicolumn{5}{|l|}{ Não trabalha } \\
\hline NIF > NV & 12,9 & 24,4 & 1,00 & $1,61-2,94$ \\
\hline $\mathrm{NIF} \leq \mathrm{NV}$ & 87,1 & 75,6 & 2,17 & \\
\hline $\mathrm{n}$ & 504 & 1.199 & & \\
\hline \multicolumn{5}{|l|}{ Trabalha } \\
\hline NIF > NV & 13,4 & 28,7 & 1,00 & $1,96-3,46$ \\
\hline $\mathrm{NIF} \leq \mathrm{NV}$ & 86,6 & 71,3 & 2,60 & \\
\hline $\mathrm{n}$ & 508 & 1.667 & & \\
\hline Renda familiar ${ }^{\star \star}$ & & & 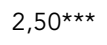 & $2,04-3,13$ \\
\hline \multicolumn{5}{|l|}{ Até $\mathrm{R} \$ 800,00$} \\
\hline $\mathrm{NIF}>\mathrm{NV}$ & 14,2 & 24,6 & 1,00 & $1,41-2,75$ \\
\hline$N I F \leq N V$ & 85,8 & 75,4 & 1,97 & \\
\hline $\mathrm{n}$ & 351 & 1.470 & & \\
\hline \multicolumn{5}{|l|}{$>\mathrm{R} \$ 800,00$} \\
\hline NIF > NV & 12,4 & 29,7 & 1,00 & $2,24-3,95$ \\
\hline$N I F \leq N V$ & 87,6 & 70,3 & 2,97 & \\
\hline $\mathrm{n}$ & 587 & 1.192 & & \\
\hline n total & 1.012 & 2.866 & & \\
\hline
\end{tabular}

*Falta informação de uma mulher; ** faltam informações de 278 mulheres, corte feto pela mediana; *** odds ratio de Mantel-Haenszel; $\mathrm{n}=$ número de mulheres. 
Distribuição percentual das mulheres segundo categorias de número ideal de filhos (NIF)

versus número de nascidos vivos (NV), de acordo com características reprodutivas.

\begin{tabular}{|c|c|c|c|c|}
\hline NIF versus NV & Laqueadas (\%) & Não-laqueadas (\%) & $O R^{\star \star}$ & IC $95 \%$ por OR \\
\hline Número de gravidezes* & & & 2,06 ** & $1,67-2,54$ \\
\hline \multicolumn{5}{|l|}{ Até 2} \\
\hline $\mathrm{NIF}>\mathrm{NV}$ & 17,1 & 33,9 & 1,00 & $1,91-3,25$ \\
\hline $\mathrm{NIF} \leq \mathrm{NV}$ & 82,9 & 66,1 & 2,49 & \\
\hline $\mathrm{n}$ & 474 & 1.890 & & \\
\hline \multicolumn{5}{|l|}{$>2$} \\
\hline $\mathrm{NIF}>\mathrm{NV}$ & 9,7 & 13,2 & 1,00 & $1,00-2,03$ \\
\hline $\mathrm{NIF} \leq \mathrm{NV}$ & 90,3 & 86,8 & 1,42 & \\
\hline $\mathrm{n}$ & 538 & 976 & & \\
\hline Número de partos & & & 2,03 ** & $1,65-2,51$ \\
\hline \multicolumn{5}{|l|}{ Até 2} \\
\hline $\mathrm{NIF}>\mathrm{NV}$ & 16,9 & 32,2 & 1,00 & $1,85-2,94$ \\
\hline $\mathrm{NIF} \leq \mathrm{NV}$ & 83,1 & 67,8 & 2,33 & \\
\hline $\mathrm{n}$ & 632 & 2.278 & & \\
\hline \multicolumn{5}{|l|}{$>2$} \\
\hline $\mathrm{NIF}>\mathrm{NV}$ & 6,8 & 6,1 & 1,00 & $0,51-1,54$ \\
\hline $\mathrm{NIF} \leq \mathrm{NV}$ & 93,2 & 93,9 & 0,89 & \\
\hline $\mathrm{n}$ & 380 & 588 & & \\
\hline Número de abortos & & & 2,43 ** & $1,98-2,98$ \\
\hline \multicolumn{5}{|l|}{ Nenhum } \\
\hline $\mathrm{NIF}>\mathrm{NV}$ & 12,7 & 26,9 & 1,00 & $1,99-3,20$ \\
\hline $\mathrm{NIF} \leq \mathrm{NV}$ & 87,3 & 73,1 & 2,52 & \\
\hline $\mathrm{n}$ & 771 & 2.229 & & \\
\hline \multicolumn{5}{|l|}{$\geq 1$ aborto } \\
\hline $\mathrm{NIF}>\mathrm{NV}$ & 14,5 & 26,8 & 1,00 & $1,42-3,29$ \\
\hline $\mathrm{NIF} \leq \mathrm{NV}$ & 85,5 & 73,2 & 2,16 & \\
\hline $\mathrm{n}$ & 241 & 637 & & \\
\hline Nascidos mortos & & & 2,43 ** & $1,98-2,98$ \\
\hline \multicolumn{5}{|l|}{ Nenhum } \\
\hline NIF $>$ NV & 13,1 & 26,8 & 1,00 & $1,97-2,98$ \\
\hline$N I F \leq N V$ & 86,9 & 73,2 & 2,42 & \\
\hline $\mathrm{n}$ & 1.000 & 2.836 & & \\
\hline \multicolumn{5}{|l|}{$\geq 1$ nascido morto } \\
\hline NIF $>N V$ & 16,7 & 36,7 & 1,00 & $0,45-23,3$ \\
\hline $\mathrm{NIF} \leq \mathrm{NV}$ & 83,3 & 63,3 & 2,89 & \\
\hline $\mathrm{n}$ & 12 & 30 & & \\
\hline $\mathrm{n}$ total & 1.012 & 2.866 & & \\
\hline
\end{tabular}

* Corte feito pela mediana; ** odds ratio de Mantel-Haenszel; $n$ = número de mulheres.

também foi observado, por exemplo, por Faúndes et al. 24. É possível que a associação entre escolaridade e risco para laqueadura tenha sido encoberta pela associação com a renda, inferindo-se que as mulheres que declararam maior renda eram também as de maior escolaridade. A propósito da renda como fator de risco, deve-se lembrar que, no período em que as mulheres entrevistadas estiveram expostas ao risco de laqueadura (1985-1995), a obtenção da esterilização ocorria, freqüentemente, mediante pagamento adicional, "por fora”, independente de ser realizada por serviços públicos ou privados $11,22,23,25,26$.
Vale a pena assinalar também que o trabalho remunerado comportou-se como fator de proteção à realização da laqueadura nesse grupo de mulheres com NIF $\leq \mathrm{NV}$, o que poderia ser visto como uma contradição, uma vez que, com freqüência, se tem estabelecido uma relação entre a opção pela laqueadura e a necessidade das mulheres se inserirem no mercado de trabalho 27,28,29. Porém, também é possível discutir que a relação entre laqueadura e trabalho remunerado ocorra de maneira distinta: que as mulheres para as quais o trabalho remunerado ocupa, ou passa a ocupar, uma posição central em seu projeto de vida, ao lado da maternida- 
Variáveis associadas à realização de laqueadura, por análise de regressão logística múltipla $(n=3.599)$.

\begin{tabular}{|c|c|c|c|c|c|}
\hline Variável & Coeficiente & EP do coeficiente & $p$ & OR & IC95\% por OR \\
\hline Idade (anos) & 0,11 & 0,01 & $<0,001$ & 1,11 & $1,09-1,13$ \\
\hline Renda familiar (> R\$ 800,00) & 0,73 & 0,09 & $<0,001$ & 2,07 & $1,74-2,46$ \\
\hline NIF versus NV (NIF $\leq N V)$ & 0,75 & 0,12 & $<0,001$ & 2,12 & $1,69-2,66$ \\
\hline Estado marital (com companheiro) & 0,76 & 0,15 & $<0,001$ & 2,14 & $1,59-2,88$ \\
\hline Número de gravidezes $(>2)$ & 0,82 & 0,18 & $<0,001$ & 2,27 & $1,61-3,20$ \\
\hline Número de abortos $(\geq 1)$ & $-0,49$ & 0,14 & $<0,001$ & 0,61 & $0,46-0,81$ \\
\hline Número de partos (> 2) & 0,56 & 0,17 & $<0,001$ & 1,75 & $1,26-2,42$ \\
\hline Trabalha (sim) & $-0,26$ & 0,08 & 0,002 & 0,77 & $0,65-0,91$ \\
\hline Constante & $-6,45$ & 0,37 & $<0,001$ & & \\
\hline
\end{tabular}

$E R=$ erro padrão

$\mathrm{OR}=$ odds ratio

de, adquirem maior autonomia quanto às decisões reprodutivas, e sentem-se mais capazes de regular sua fecundidade, sem chegar, necessariamente, a ponto de não ter outra opção que não seja a laqueadura 15 .

Finalmente, a presença de um ou mais abortamentos também se apresentou como fator de proteção para a laqueadura, o que poderia indicar que uma parte das mulheres com NIF $\leq$ NV poderia ter recorrido ao aborto para evitar gestações indesejadas, embora no Brasil essa prática não seja legal 1,3.

Cabe salientar que o fato de se tratar de uma análise secundária, onde o desenho original de estudo não contemplava este tipo de abordagem, de certa forma limita os resultados apresentados. Além disso, nos estudos de caso-controle quando a prevalência da exposição (NIF $\leq$ NV) é superior a $25,0 \%$, existe uma tendência a se superestimar os riscos estimados. Adicionalmente, este método epidemiológico quantitativo pode apenas explicar uma parcela dos resultados encontrados, visto que a realidade das mulheres com relação ao desejo pelo número de filhos e à laqueadura está ainda intimamente relacionada com aspectos sociais, psicológicos e culturais que apenas uma abordagem qualitativa complementar poderia melhor completar esse enfoque analítico.
Entretanto, os achados deste estudo corroboram com a discussão que já tem estado presente na literatura da área quanto às dificuldades que as mulheres enfrentam para regular sua fecundidade, o que inclui a falta de acesso à informação e aos métodos anticoncepcionais, passando pelo não planejamento de sua vida reprodutiva no contexto de um projeto de vida mais amplo 10,12,22,27,30. Nossos achados iluminam ainda mais essa discussão, pois permitem visualizar que a opção pela laqueadura não se dá em função das mulheres atingirem seu NIF, pois freqüentemente esse número já havia sido ultrapassado. Mais uma vez evidencia-se a necessidade de prover acesso amplo aos meios para a regulação da fecundidade, visando a assegurar que as pessoas possam, de fato, decidir sobre quando e quantos filhos ter, um dos elementos fundamentais do conceito de saúde reprodutiva. Entende-se que essa provisão seja um objetivo fundamental da Lei Sobre Planejamento Familiar, que passou a vigorar a partir de 1996 (Portaria no 144. Diário Oficial da União 1997; 24 nov). Porém, até este momento não se dispõe de avaliações que permitam saber se, desde então, houve melhora no panorama da anticoncepção em nosso país. Esta é uma tarefa imperativa no sentido de prover atendimento de qualidade à saúde reprodutiva das mulheres brasileiras. 


\section{Resumo}

O objetivo deste artigo é avaliar a associação entre o número ideal de filhos (NIF) e a realização da laqueadura. Foi feito um estudo de caso-controle aninhado, com base em uma análise secundária de dados obtidos em um estudo de coorte sobre a saúde reprodutiva de mulheres em Campinas, São Paulo, Brasil, que teve como sujeitos 3.878 mulheres, das quais 1.012 eram laqueadas (casos). A relação entre NIF e o número de nascidos vivos (NV) foi dividida em duas categorias $(N I F>N V$ e NIF $\leq N V)$. Foram calculados os riscos relativos estimados (odds ratio) para a realização de laqueadura e seus respectivos intervalos de confiança (IC) 95\% para a relação NIF/NV, estratificando-se para outras dez variáveis. Todas as variáveis preditoras foram incluídas em um modelo de regressão logística para identificar os fatores independentemente associados à esterilização definitiva. Observou-se que o risco de laqueadura foi maior entre as mulheres com $N I F \leq N V$, maior idade, maior renda familiar, com companheiro, com mais de duas gestações, maior número de partos, com menor número de abortos e sem trabalho remunerado.

Métodos Anticoncepcionais; Laqueadura Tubária; Planejamento Familiar; Controle da Natalidade

\section{Colaboradores}

J. G. Cecatti elaborou e escreveu o projeto original do estudo de coorte de onde deriva o presente enfoque. M. J. D. Osis e J. G. Cecatti participaram de todas as fases da pesquisa e, conjuntamente com L. E. C. Carvalho e M. H. Souza, planejaram sua abordagem analítica. L. E. C. Carvalho e M. H. Souza realizaram a análise estatística, conferência e discussão dos resultados, montagem das tabelas, e escreveram a primeira versão do artigo. Todos participaram da discussão e redação da versão final do artigo.

\section{Referências}

1. Bongaarts J. Trends in unwanted childbearing in the developing world. Stud Fam Plann 1997; 28: 267-77.

2. Perpétuo IHO, Aguirre MACO. Papel da esterilização feminina nos diferenciais socioeconômicos do declínio da fecundidade no Brasil. In: Anais do XI Encontro Nacional de Estudos Populacionais; 1998 Out 19-23; Caxambu, Minas Gerais. Campinas: Associação Brasileira de Estudos Populacionais; 1998. p. 2997-3023.

3. Martine G. Brazil's fertility decline, 1965-95: a fresh look at key factors. Popul Dev Rev 1996; 22:47-75.

4. Harbison SF, Robinson WC. Policy implications of the next demographic transition. Stud Fam Plann 2002; 33:37-48.

5. Faria VE. Políticas de governo e regulação de fecundidade: conseqüências não antecipadas e efeitos perversos. Ciências Sociais Hoje 1989; 62-103.

6. Osis MJD. Paism: um marco na abordagem da saúde reprodutiva no Brasil. Cad Saúde Pública 1998; 14 Suppl 1:25-32.

7. Schor N, Ferreira AF, Machado VL, França AP, Pirotta KCM, Alvarenga AT, et al. Mulher e anticoncepção: conhecimento e uso de métodos anticoncepcionais. Cad Saúde Pública 2000; 16:377-84.

8. Sociedade Civil Bem-Estar Familiar no Brasil. Pesquisa Nacional sobre Demografia e Saúde, 1996. Rio de Janeiro: Sociedade Civil Bem-Estar Familiar no Brasil/Macro Internacional Inc.; 1997.

9. Barbosa CP, Pellini, EAJ, Reis AV, Sato M, Lesser R, Marques RS, et al. Avaliação do grau de insatisfação pós-laqueadura em São Bernardo do Campo. Reprod Cilm 1994; 9:159-62.

10. Minella LS. Aspectos positivos e negativos da esterilização tubária do ponto de vista de mulheres esterilizadas. Cad Saúde Pública 1998; 14 Suppl 1:69-79.

11. Vieira EM. O arrependimento após a esterilização feminina. Cad Saúde Pública 1998; 14 Suppl 1:5968.

12. Osis MJD, Faúndes A, Sousa MH, Bailey P. Conseqüências do uso de métodos anticoncepcionais na vida das mulheres: o caso da laqueadura tubária. Cad Saúde Pública 1999; 15:521-32.

13. Osis MJD, Faúndes A, Sousa MH, Duarte GA, Bailey P. Fertility and reproductive history of sterilized and non-sterilized women in Campinas, São Paulo, Brazil. Cad Saúde Pública 2003; 19:1399404.

14. Osis MJD, Faúndes A, Sousa MH, Bailey P, Duarte G, Souza TR. Which characteristics differentiate women of the same age who are sterilized from those who are not? In: Proceedings of the X World Congress on Human Reproduction; 1999 Mai 4-8; Salvador, Bahia. Bologna: International Proceedings Division; 1999. p. 211-4.

15. Osis MJD. Laqueadura e representações acerca da sexualidade e do papel reprodutivo [Tese de Doutorado]. São Paulo: Faculdade de Saúde Pública, Universidade de São Paulo; 2001.

16. Jejeebhoy SJ. Cohort consistency in family size preferences: Taiwan, 1965-73. Stud Fam Plann 1981; 12:229-32.

17. Herrera EZ. Cambios en el nivel de la fecundidad 
deseada en las mujeres mexicanas, 1967-1986. Rev Mex Sociol 1993; 55:83-97.

18. Arruda JM, Rutenberg N, Morris L, Ferraz EA. Pesquisa Nacional Sobre Saúde Materno-Infantil e Planejamento Familiar: Brasil, 1986. Rio de Janeiro: Sociedade Civil Bem-Estar Familiar no Brasil/Instituto para Desenvolvimento de Recursos; 1987.

19. Pires HMB. O impacto das altas taxas de cesárea sobre a fecundidade de uma população. Um estudo de coorte retrospectivo em Campinas, Brasil [Tese de Doutorado]. Campinas: Faculdade de Ciências Médicas, Universidade Estadual de Campinas; 2000.

20. Schlesselman JJ. Case-control studies. Design, conduct, analysis. New York: Oxford University Press; 1982.

21. Bongaarts J. The measurement of wanted fertility. Popul Dev Rev 1990; 16:487-506.

22. Vieira EM. A esterilização de mulheres de baixa renda em região metropolitana do sudeste do Brasil e fatores ligados à sua prevalência. Rev Saúde Pública 1994; 28:440-8.

23. Vieira EM, Ford NJ. The provision of female sterilization in São Paulo, Brasil: a study among lowincome women. Soc Sci Med 1996; 42:1427-32.

24. Faúndes A, Costa RG, Pádua KS, Perdigão AM. Associação entre prevalência da laqueadura tubária e características sociodemográficas de mulheres e seus companheiros no Estado de São Paulo. Cad Saúde Pública 1998; 14 Suppl 1:49-57.
25. Osis MJD, Hardy E, Simões IR, Vera S, Faúndes A. Laqueadura tubária nos serviços de saúde do Estado de São Paulo. Rev Ginecol Obstet 1991; 1:195204.

26. Giffin K. Women's health and the privatization of fertility control in Brazil. Soc Sci Med 1994; 39:35560.

27. Marcolino C. Trajetória da mulher em direção à esterilização cirúrgica feminina: um estudo fenomenológico [Dissertação de Mestrado]. São Paulo: Faculdade de Saúde Pública, Universidade de São Paulo; 1994.

28. Costa RG, Osis MJD, Hardy E. Considerações sobre o processo de decisão pela laqueadura. In: Anais do X Encontro Nacional de Estudos Populacionais; 1996 Out 7-11; Caxambu, Minas Gerais. Campinas: Associação Brasileira de Estudos Populacionais; 1996. p. 2523-35.

29. Serruya S. Ligação de trompas e imaginário feminino. Rev Bras Estud Popul 1993; 10:57-70.

30. Espejo X, Tsunechiro MA, Osis MJD, Duarte GA, Bahamondes L, Sousa MH. Adequação do conhecimento sobre métodos anticoncepcionais entre mulheres de Campinas, SP, Brasil. Rev Saúde Pública 2003; 37:583-90.

Recebido em 22/Set/2003

Versão final reapresentada em 20/Abr/2004

Aprovado em 21/Jun/2004 